\title{
The MAD View on the Outskirts of Disks
}

\author{
C. M. Carollo, S. Erroz-Ferrer, M. den Brok, M. Fagioli, \\ M. Onodera, S. Tacchella and the MAD collaboration
}

\author{
Department of Physics, Institute for Astronomy, ETH Zurich, CH-8093 Zurich, Switzerland \\ email: marcella@phys.ethz.ch
}

\begin{abstract}
We present the MUSE Atlas of Disks (MAD), a GTO program with the MUSE spectrograph on the ESO/VLT that is dedicated to the study of the optical spectroscopic properties of $z=0$ disk galaxies on the star-forming 'Main Sequence' at $<100$ pc physical resolution. MUSE pointings on the MAD galaxies extend out to $\sim 2$ disk scale lengths, enabling to investigate the bulge and inner disk properties of galaxies with different outer disks. Here we specifically compare, at constant stellar-mass, the stellar population properties of the inner components in disks with down-bending (Type II) and up-bending (Type III) outer profiles. We highlight similarities in the inner stellar properties of such different hosts, which point at a universal inside-out growth of disks, as well as differences which suggest an additional role of stellar migration and/or gas accretion in the growth of disk galaxies with an outer up-bending profile, as expected from theoretical models.
\end{abstract}

Keywords. galaxies: spiral - galaxies: structure - galaxies: evolution

\section{Introduction}

Over the past decades we have learned key scaling laws that link the integrated properties of star-forming galaxies. At any epoch these galaxies grow in mass and size along a Main Sequence in which the SFR, normalized to the cosmic average (e.g., Madau et al. 1996; Lilly et al. 1996; Madau \& Dickinson 2014), is proportional to the existing galaxy stellar mass, $\mathrm{M}_{*}$ (e.g., Brinchmann et al. 2004; Daddi et al. 2007; Noeske et al. 2007). Also, typical Main Sequence galaxies appear to have a disk morphology, possibly also at early epochs (e.g., Bell et al. 2012; Genzel et al. 2008) but certainly at $z=0$ (e.g., Strateva et al. 2001).

On small spatially-resolved scales, the Hubble Space Telescope has at the same time produced a wealth of optical and near-IR images of nearby star-forming disks at $<100$ pc resolution (e.g., Malkan et al. 1998; Martini \& Pogge 1999; Carollo et al. 1997, 1998, 2001, 2002; Böker et al. 2002, 2004; Seth et al. 2008). These data show that typical $10^{8}-10^{11} M_{\odot}$ Main Sequence disks host substantial bulges which have however low n-Sérsic indices (e.g., Courteau et al. 1996; Carollo et al. 1998).

The disk-like bulges of $z=0$ Main Sequence galaxies can be as old as the Milky Way Bulge (Carollo et al. 2007). From optical colors, however, it would appear that these bulges often feature relatively young stellar populations, indicating that they are still under construction today (e.g., Kormendy \& Kennicutt 2004). Ongoing bulge formation inside Main Sequence disks would not be unexpected, since they are thought to be still accreting relatively cold gas from narrow streams (Keres et al. 2005; Dekel et al. 2009; Agertz et al. 2009; Danovich et al. 2012). Furthermore it has been shown that gas inflows towards the centers of disks could grow bulge components (e.g., Shlosman \& Noguchi 1993; Combes 2008); bar structures may be one of the causes behind gas inflow and bulge formation (e.g., Norman et al. 1996; Combes 1990; Debattista et al. 2006). 
Accurate spectroscopic determination of the stellar ages of the innermost disk regions is crucial to discriminate amongst different theoretical ideas.

Spectroscopic diagnostics at high angular resolution are also awaited to understand how disks sustain their global Main Sequence 'equilibrium' (e.g., Genel et al. 2008; Bouché et al. 2010; Schaye et al. 2010; Davé et al. 2012; Krumholz \& Dekel 2012; Lilly et al. 2013; Tacchella et al. 2016), and also what the local conditions within individual disks which determine the growth of stellar mass in their inner bulge regions are, as galaxies as a whole keep moving along the Main Sequence with cosmic time. Key facts are indeed still unknown, i.e., whether and how strongly SFR (and stellar feedback) depend on the local stellar mass density, how this depends on stellar metallicity and/or age, whether ionized gas properties (density, temperature, metallicity) also modulate the SFR vs. stellar mass density relation; what conditions - local/global winds, shocks, AGN - halt or stimulate local star formation; how heavy elements are redistributed across the inner galactic regions; whether local or global gas outflows are relevant in building up central structure in disks - as galaxies as a whole keep 'peacefully' evolving along the Main Sequence.

A new avenue of study is indeed being opened by 2-dimensional spectroscopy, which enables spatially-resolved chemo-dynamical maps of nearby disks. DiskMass (Bershady et al. 2010), CALIFA (Sánchez et al. 2012), MaNGA (Bundy et al. 2015), SAMI (Bryant et al. 2015) are returning such maps, for large statistical samples of several thousand galaxies, resolved on scales of 1-to-few kpc. Full complementary to these surveys, thanks to the capabilities of MUSE (Bacon et al. 2010) and to our sample selection criteria, our MUSE Atlas of Disks (MAD; Carollo et al. 2016, in preparation, hereafter Paper I) program of 2-dimensional optical spectroscopy with MUSE on the ESO/VLT is rather uniquely probing the centers of $z=0$ star-forming Main Sequence disks at a physical resolution of $<100 \mathrm{pc}$. These are the (small) scales of relevance for studying the physics involved in the formation and evolution of disk galaxies and their structural subcomponents.

\section{MAD dissection at $<100$ pc resolution of the cores of $z=0$ disks}

MUSE is a second-generation instrument mounted on the ESO VLT. It is an optical (4700-9500 $\AA$ ) Integral-Field Spectrograph with a field-of-view of $1 \times 1$ arcmin $^{2}$ in Wide-Field Mode. The spatial sampling is 0.2 arcsec; the spectral resolution varies from 2500 in the blue to 4000 in the red. Working in seeing-limited mode at the time of writing, MUSE will use Adaptive Optics (AO) technology starting early-2017.

The full MAD sample will contain about 100 nearby galaxies with stellar masses between $10^{8}$ and $10^{11} M_{\odot}$, delineating the star-forming Main Sequence at $z \approx 0$ (see Fig 1), i.e., aligning along the $\log \mathrm{SFR}>-7.74+0.69 \times \log M_{\star}$ relation (Renzini et al. 2015). The MAD galaxies lie within $z<0.012$, so as to ensure a resolution of $<100 \mathrm{pc}$; at least one and in some cases two disk scale lengths are covered with a single MUSE pointing (centered on the galaxies' nuclei). All MAD galaxies have HST imaging in at least one optical band. By the time of writing this article, about a quarter of the total sample, i.e., 25 MAD galaxies, have already been observed and analyzed. These galaxies are highlighted with large red points in Fig 1. They are representative of the full MAD sample.

For all MAD galaxies, MUSE returns $(i)$ the suit of emission lines from $\mathrm{H} \beta$ to $[\mathrm{SII}] 6731 \AA$ that enable the study of the physical properties and kinematics of the ionized gas including extinction maps, metallicity, dust-corrected SFR maps and distributions of very young, $<20$ Myr-old stellar populations; ( $i i$ ) all important absorption line 


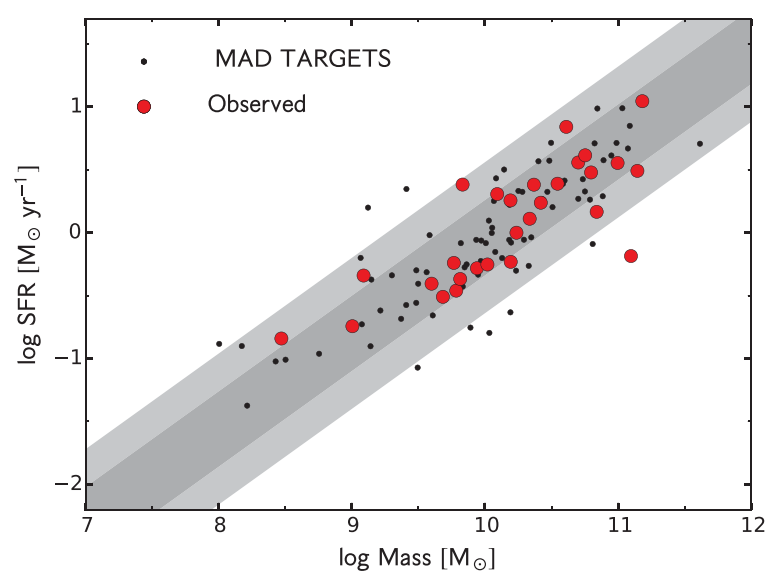

Figure 1. The MAD sample plotted on the $z=0$ star-forming Main Sequence from Renzini et al. 2015 (black line). The grey shaded area shows its typical scatter $(1 \sigma=0.3 \mathrm{dex}$, dark; also highlighted in lighter grey are the $2 \sigma$ strips). The black and large red points show the full MAD sample and the 25 already observed MAD galaxies, respectively.

diagnostics from the age-sensitive $\mathrm{H} \beta$ at the blue-end of the MUSE spectra, to the $\sim 5000$ $\AA \mathrm{Mg}$ and Fe lines, out to the CaII lines at $8500 \AA$. These provide superb stellar kinematics as well as key constraints to stellar populations properties, i.e., stellar mass density and (luminosity- and mass-weighted) stellar ages and metallicities.

The 2-dimensional MAD maps of stellar and gaseous kinematics and absorption/emission line properties (stellar populations, gas-phase metallicities, dust extinction, SFRs, ISM properties) achieve much higher spatial resolution, denser sampling and greater depth than any other currently ongoing program of 2-dimensional spectroscopy on such systems. This unique data set enables us to detect gas in/outflows, shocks, kinematic and chemical/population signatures of accretion events -and also, particularly relevant to the following discussion, the dynamical state of inner disks and bulges and their star-formation histories in galaxies with known and diverse outer disk profiles.

\section{Classification of MAD galaxies from their outer-disk shapes}

The spatial coverage of the MAD galaxies is tailored to investigate their bulges, inner disks and other nuclear substructures that are easily identified in optical images as light in excess of an inward extrapolation of a single-exponential disk. Indeed, over a radial range of several disk scale lengths, the light profiles of star-forming disks are described by an exponential profile. Structure is also clearly evident however also in the galactic peripheries, in particular as breaks in the outward exponential profiles (e.g., Pohlen \& Trujillo 2006, Laine et al. 2014, Marino et al. 2016). The radial location of these breaks moves further away from the galaxies' centers with passing cosmic time (Perez 2004; Azzollini et al. 2008). More than two thirds of disks show such breaks.

Disk galaxies have been classified according to their light profiles (Erwin et al. 2005; Pohlen \& Trujillo 2006) as Type I (no break), Type II (down-bending break) and Type III (up-bending break). Several theoretical ideas have been put through to explain the origin of these differences in the outer disk profiles. To mention a few, Type II breaks have been proposed to arise due to, e.g., a redistribution of angular momentum (e.g., van der Kruit 1987), a star formation threshold (e.g., Kennicutt 1989) or stellar migration (e.g., Roškar et al. 2008a, 2008b, 2010, Sánchez-Blázquez et al. 2014). In contrast, mergers, 
Table 1. The global properties of the MAD galaxies that we study in this paper. The top seven and the bottom six are classified respectively as Type II and Type III according to the shape of their outer surface brightness profiles. The columns in the table indicate, from left to right: Galaxy name, distance in Mpc, stellar mass in $M_{\odot}$, SFR (from SED fits) in $M_{\odot} \mathrm{yr}^{-1}$, half-light radius $R_{\mathrm{e}}$ in $\mathrm{kpc}$, break radius in $\mathrm{kpc}$. The last column lists the data used to derive the surface brightness profiles (SDSS, CGS or $\mathrm{S}^{4} \mathrm{G}$ ) and measure their outer breaks.

\begin{tabular}{|c|c|c|c|c|c|c|}
\hline Galaxy & $\begin{array}{c}\mathrm{D} \\
(\mathrm{Mpc})\end{array}$ & $\begin{array}{c}M_{\star} \\
\left(M_{\odot}\right)\end{array}$ & $\begin{array}{c}\mathrm{SFR} \\
\left(M_{\odot} \mathrm{yr}^{-1}\right)\end{array}$ & $\begin{array}{c}R_{\mathrm{e}} \\
(\mathrm{kpc})\end{array}$ & $\begin{array}{r}R_{\text {break }} \\
(\mathrm{kpc})\end{array}$ & Source Data \\
\hline \multicolumn{7}{|l|}{ Type II } \\
\hline NGC 1042 & 15.0 & $6.79 \mathrm{E}+09$ & 2.41 & 4.7 & 7.3 & SDSS, CGS, $\mathrm{S}^{4} \mathrm{G}$ \\
\hline NGC 4603 & 32.8 & $1.25 \mathrm{E}+11$ & 0.65 & 7.2 & 13.2 & CGS \\
\hline NGC 4900 & 21.6 & $1.72 \mathrm{E}+10$ & 1.00 & 3.5 & 8.6 & $\mathrm{~S}^{4} \mathrm{G}$ \\
\hline NGC 4941 & 21.2 & $6.26 \mathrm{E}+10$ & 3.01 & 4.8 & 7.4 & CGS, $\mathrm{S}^{4} \mathrm{G}$ \\
\hline NGC 5643 & 17.4 & $6.90 \mathrm{E}+10$ & 1.46 & 5.1 & 10.8 & CGS \\
\hline NGC 7421 & 25.4 & $1.24 \mathrm{E}+10$ & 2.03 & 3.7 & 6.2 & CGS, $S^{4}$ G \\
\hline NGC 7552 & 14.8 & $1.55 \mathrm{E}+10$ & 1.80 & 1.9 & 6.7 & CGS, $S^{4} G$ \\
\hline \multicolumn{7}{|l|}{ Type III } \\
\hline NGC 289 & 24.8 & $9.91 \mathrm{E}+10$ & 3.58 & 3.2 & 9.0 & CGS, $\mathrm{S}^{4} \mathrm{G}$ \\
\hline NGC 1309 & 31.2 & $2.33 \mathrm{E}+10$ & 2.41 & 3.0 & 10.3 & CGS, $S^{4} G$ \\
\hline NGC 3256 & 38.4 & $1.40 \mathrm{E}+11$ & 3.10 & 5.0 & 9.1 & CGS \\
\hline NGC 4030 & 29.9 & $1.52 \mathrm{E}+11$ & 11.08 & 4.6 & 20.6 & SDSS, $S^{4} G$ \\
\hline NGC 5806 & 26.8 & $5.02 \mathrm{E}+10$ & 3.61 & 3.5 & 6.9 & SDSS, $S^{4} G$ \\
\hline NGC 7496 & 11.9 & $1.56 \mathrm{E}+10$ & 0.59 & 3.9 & 3.9 & CGS, $S^{4} G$ \\
\hline
\end{tabular}

interactions or gas accretion are thought to be one of the most plausible causes of Type III breaks (e.g., Laurikainen \& Salo 2001, Gil de Paz et al. 2005, Minchev et al. 2012).

Different scenarios for the build up of outer breaks carry implications for the growth and properties of galaxy centers. Viceversa, we can look at the properties of the central regions of disks - their bulges, inner disks and other nuclear features - in order to place constraints to scenarios of outer-disk, and in general, disk galaxy formation. Here we take the latter perspective and group the currently available MAD galaxies according to their outer-profile classification, so as to investigate whether the MAD spectroscopic maps of the central regions also show substantial differences as the galaxies' outskirts do.

Extended surface brightness profiles are available from the literature for several MAD galaxies, in particular from $B, V, R$ and $I$ images of the Carnegie-Irvine Galaxy Survey (CGS; Ho et al. 2011) and $3.6 \mu \mathrm{m}$ images of the Spitzer Survey for Stellar Structure in Galaxies ( $\mathrm{S}^{4} \mathrm{G}$; Sheth et al. 2010). Specifically, surface brightness profiles based on CGS and $\mathrm{S}^{4} \mathrm{G}$ photometry are published in Zhao-Yu et al. (2011) and Muñoz-Mateos et al. (2015), respectively. For the remaining galaxies in our current MAD sample we derived their surface brightness from $g^{\prime}$-band SDSS data from DR12 (Alam et al. 2015) following the methodology detailed in Pohlen \& Trujillo (2006). We then fitted two exponential profiles to the inner and outer disks of our galaxies in order to identify, at the intersection of the two, the location of their break radii (listed in Table 1). For illustration, Fig. 2 shows the surface brightness profiles of two of our galaxies, specifically a galaxy example of Type II (NGC 1042) and one of Type III (NGC 1309) break.

In the following we focus on comparing Type II and Type III galaxies, as there are barely any Type I systems in our MAD sample with currently available MUSE data; this paucity of Type Is is expected from the known frequency of occurrence of each of the 

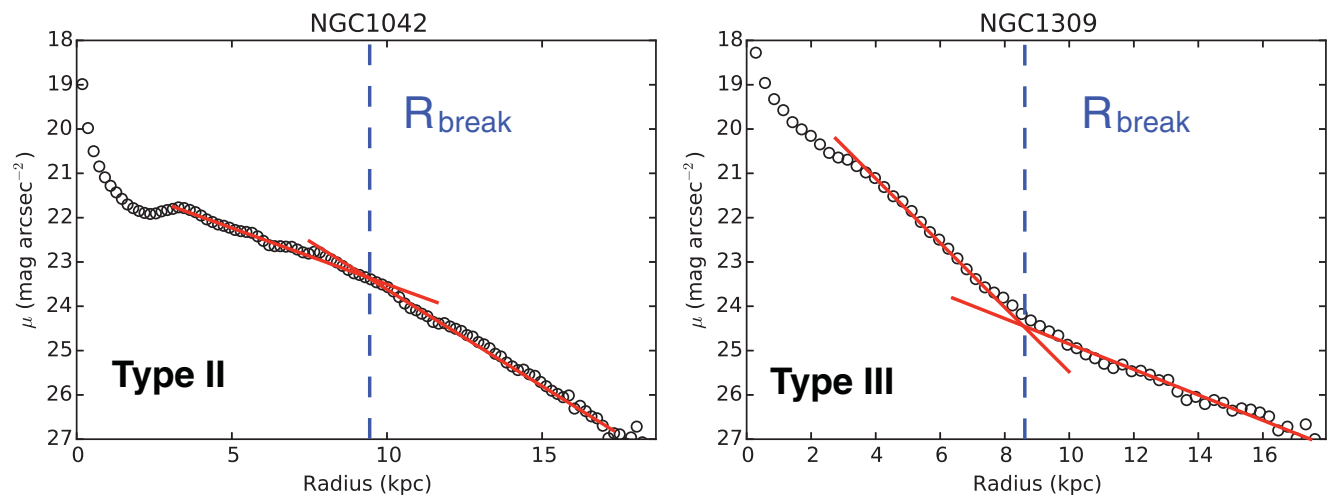

Figure 2. Surface brightness profiles of two MAD galaxies, namely NGC 1042 (Type II; left) and NGC 1309 (Type III; right). Red lines show the exponential inner and outer fits; in blue we highlight the location of their break radii.
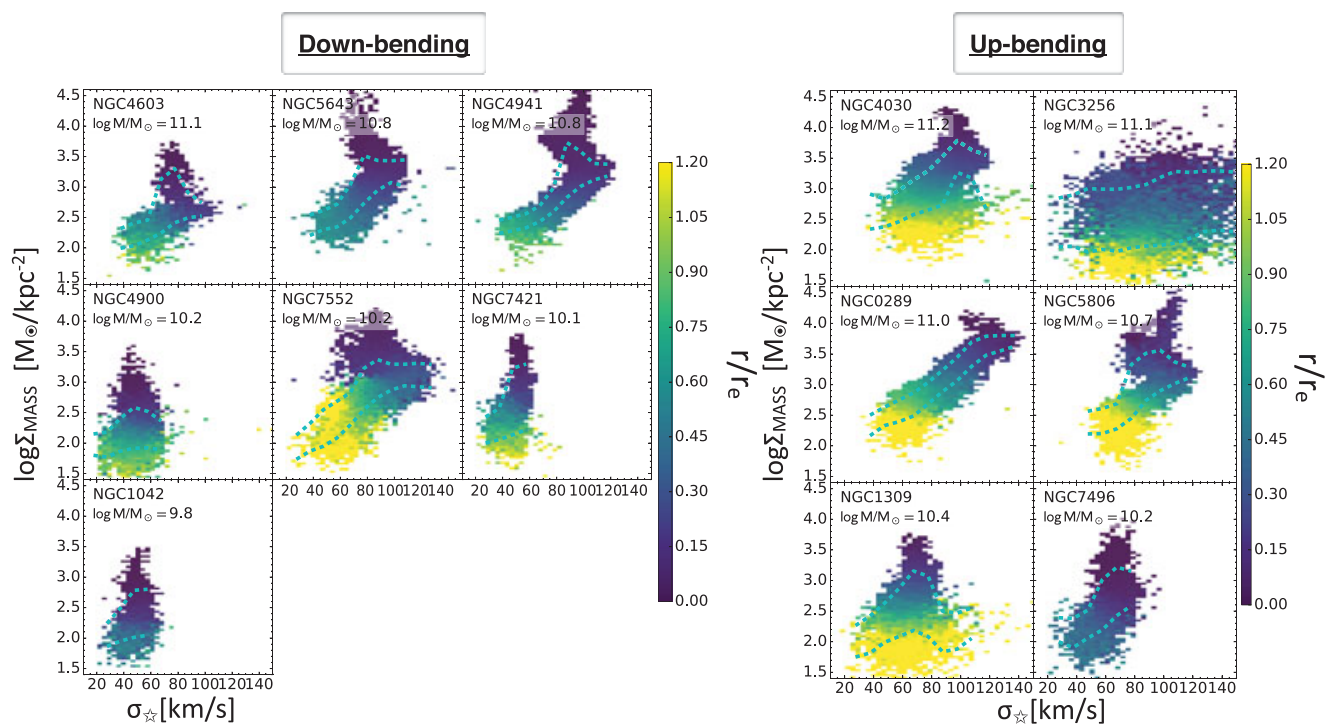

Figure 3. Stellar mass surface density $\Sigma_{M A S S}$ as a function of stellar velocity dispersion $\sigma_{\star}$, color-coded by galacto-centric distance $r$ (normalized by the galaxies' half-light radii, $r_{\mathrm{e}}$ ). Galaxies with Type II, down-bending outer profiles are plotted on the left, and galaxies with Type III, up-bending profiles on the right.

types (e.g., Pohlen \& Trujillo 2006, Laine et al. 2014, Marino et al. 2016). Specifically we select 13 MAD galaxies (out of the 25 already observed with MUSE) which enable us to match in stellar mass the Type II and Type III subsamples, and thus to compare these subsamples without worries that differences between the two may arise from major differences in stellar mass. This leaves us currently with seven Type II and six Type III galaxies with stellar masses in the $7 \times 10^{9}-2 \times 10^{11} M_{\odot}$ range. The limited size of these subsamples urges for caution to be exercised in generalising the preliminary trends that we briefly discuss below; once MAD is completed we will be able to increase our statistics by at least a factor of four, and thus be able to make statistically stronger statements on the inner properties of Type II and Type III disks. With this caveat in mind, we discuss below the inner stellar population properties of our Type II and Type III subsamples, 

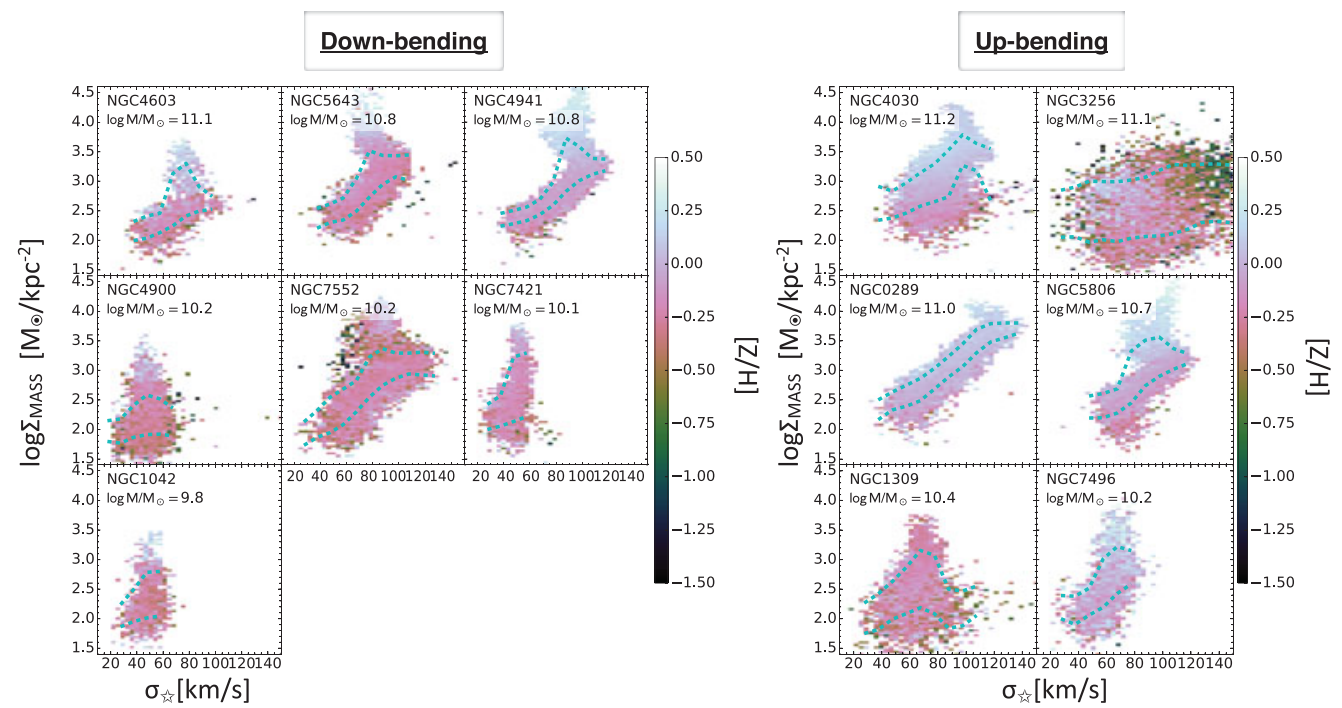

Figure 4. Stellar mass surface density $\Sigma_{M A S S}$ as a function of stellar velocity dispersion $\sigma_{\star}$, color-coded by stellar metallicity $[Z / H]$. As in Fig. 3, galaxies with Type II, down-bending outer profiles are plotted on the left, and galaxies with Type III, up-bending profiles on the right.

to infer what these imply for the global growth of the galactic disk population at late cosmic epochs.

\section{MAD stellar population properties of Type II and Type III disks}

Many questions can be addressed with our MAD data regarding the formation and accretion histories of Type II and III galaxies - many involving the analysis of the galaxies' emission line spectra (e.g., whether either, both or neither classes present direct evidence for satellite accretion, as identified through detection of low-metallicity gaseous 'streams'). An example of a MAD gas-phase analysis is mentioned in Erroz-Ferrer et al. (2016).

Here we focus however on only the inner stellar population properties of Type II and III disks, and in particular on the inter-relations between stellar mass density $\Sigma_{M A S S}$, velocity dispersion $\sigma_{\star}$, and mass-weighted stellar metallicities $([Z / H])$ and ages - of course locally-dissected within the galaxies at MAD resolution. These stellar properties are derived using the stellar population synthesis models from PEGASE-HR (Le Borgne et al. 2004), through spectral fits to the galaxies' emission-line subtracted spectra (and excluding strong emission line wavelength regions from the fits). Further details will be presented in Paper I.

Specifically, Figs. 3, 4 and 5 show, in sequential order, $\Sigma_{M A S S}$ versus $\sigma_{\star}$ colorcoded by, respectively, $r / r_{\mathrm{e}},[Z / H]$ and age. The direct comparison of surface stellar mass density with stellar velocity dispersion at different galacto-centric distances (as in Fig. 3) enables us to disentangle bulges from inner disks or, better, dense but kinematically-cold cores from genuine, kinematically-hot bulges, as well as to identify low-density, 'heated' regions of the stellar disks.

In both Type IIs and IIIs the central bulge regions look relatively similar: they are, not surprisingly, always the densest regions of the disks, although not necessarily the kinematically hottest: in many galaxies there are clear points of inflection in the 

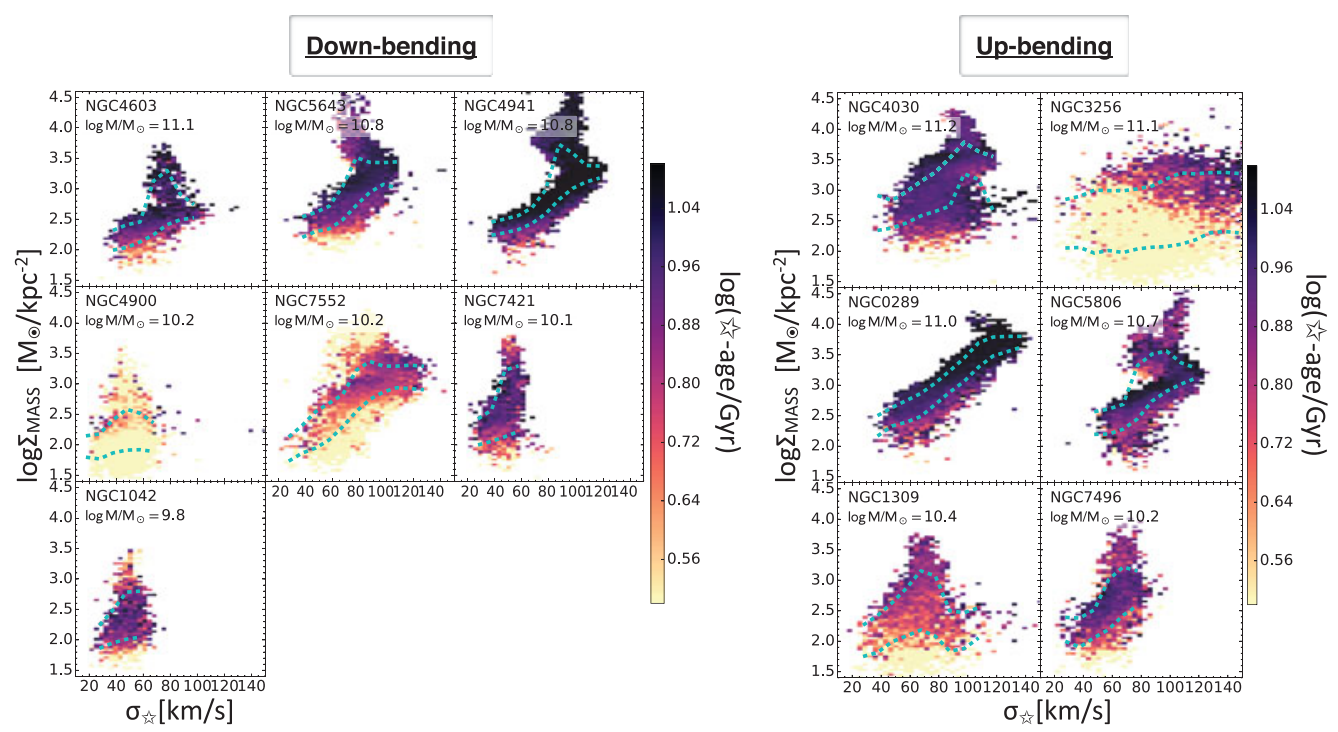

Figure 5. Stellar mass surface density $\Sigma_{M A S S}$ as a function of stellar velocity dispersion $\sigma_{\star}$, color-coded by mass-weighted stellar age. As in Figs. 3 and 4, galaxies with Type II, down-bending outer profiles are plotted on the left, and galaxies with Type III, up-bending profiles on the right.

$\Sigma_{M A S S}-\sigma_{\star}$ relation, where the innermost, densest regions turn leftward towards lower velocity dispersions relative to the adjacent disk regions.

Some differences between Type IIs and Type IIIs emerge however in their disk components. Type II galaxies tend to show well-behaved disks, with a well-defined direct proportionality between $\Sigma_{M A S S}$ and $\sigma_{\star}$. In contrast, Type IIIs can present much larger spreads in velocity dispersion than their Type II relatives, especially toward low surface mass densities. Particularly striking in this context are the examples of NGC 3256 and NGC 1309. Fig. 4 furthermore shows very flat metallicity gradients for these 'hot' Type III disks. Differently from Type IIs, which tend to show 'normal' negative gradients in metallicity, i.e., highly-enriched bulges and less enriched outer disk regions, bulges and disks in Type IIIs have similar stellar metallicities. The stellar ages (Fig. 5) in both galaxy types indicate older centres and younger outskirts, although in many galaxies there is a clear addition of younger populations also in the nuclear/bulge regions. It is interesting that the hot disks of Type III galaxies such as NGC 3256 and NGC 1309 tend to be relatively young.

\section{Concluding remarks}

MAD provides an unprecedented $<100 \mathrm{pc}$ view of the physical conditions that are typical of galaxies on the star-forming Main Sequence at $z=0$. The MUSE spectra acquired so far for one-quarter of the MAD sample give already a taste of the important knowledge that the full survey will contribute towards understanding galactic growth on the Main Sequence.

For example, already at this stage in the survey, albeit within the caveats of small sample statistics, we find interesting similarities between disks with down-bending and disks with up-bending outer radial profiles, e.g., outer, low-density disk components that are dynamically-hotter than the inner bulges. 
We also find suggestive evidence for differences in the centers of disk galaxies that differ in their outskirts. Specifically, in contrast with Type II galaxies, which generally host highly-enriched stellar components in their bulges, and systematically declining metallicity values moving outwards towards the disk outskirts (i.e., steep stellar metallicity gradients), MAD galaxies with an outer up-bending disk profile show, in the MUSE spectral diagnostics, signatures of substantial interaction of a pre-existing old disk with its cosmological surroundings, namely low-density disk regions at large galacto-centric distances that are dynamically-heated, have flat radial metallicity gradients between bulge and inner disk regions, and are clearly rejuvenated in their stellar populations. This supports scenarios of accretion of enriched material from the surroundings (Minchev et al. 2012), or possibly radial mixing (e.g., Roškar et al. 2008a). If confirmed on the total MAD sample, such differences in the inner properties of disk galaxies with different outer profiles would imply that the physical processes that shape the outer profiles have an impact down to the galaxy cores, within the central few kpc that are captured with MUSE in our MAD survey.

\section{References}

Agertz, O. et al. 2009, MNRAS, 397, 64

Alam, S. et al. 2015, ApJS, 219, 12

Azzollini, R. et al. 2008,ApJ, 679, 69

Bacon, R. et al. 2012, ApJ, 753, 167

Bell, E. et al. 2012, ApJ, 753, 167

Bershady, M. et al. 2010, ApJ, 716, 198

Böker, T. et al. 2002, AJ, 1231389

Böker, T. et al. 2004, AJ, 127, 105

Bouché, N. et al. 2010, ApJ, 718, 1001

Brinchmann, J. et al. 2004, MNRAS, 351, 1151

Bryant, J. J. et al. 2015, MNRAS, 447, 2857

Bundy, K. et al. 2015, ApJ, 798, 7

Carollo, C. M. \& Lilly, S. 2001, ApJL, 548, L153

Carollo, C. M. et al. 1997, AJ, 1142366

Carollo, C. M. et al. 1998, AJ, 116, 68

Carollo, C. M. et al. 2001, ApJ, 546, 216

Carollo, C. M. et al. 2002, AJ, 123159

Carollo, C. M. et al. 2007, ApJ, 658, 960

Combes, F. 1990, A\&AA, 233, 82

Combes, F. 2008, Proceedings of IAU Symposium 245, "Formation and evolution of Galaxy Bulges", Eds. M. Bureau, E. Athanassoula \& B. Barbury, Cambridge University Press, Cambridge., 151-160

Courteau, S. et al. 1996, ApJ, 457, 73

Daddi, E. et al. 2007, ApJ, 670, 156

Danovich, M. et al. 2012, MNRAS, 422, 1732

Davé, E. et al. 2012, MNRAS, 421, 98

Debattista, V. et al. 2006, ApJ, 645, 209

Dekel, A. et al. 2009, ApJ, 703, 785

Erroz-Ferrer, S. et al. 2016, these Proceedings of IAU Symposium 321, "Formation and evolution of galaxy outskirts", Eds. A. Gil de Paz, J. C. Lee \& J. H. Knapen, Cambridge University Press, Cambridge.

Erwin, P. et al. 2005, ApJ, 626, 81

Genel, S. et al. 2008, ApJ, 688, 789

Genzel, R. et al. 2008, ApJ, 687, 59

Gil de Paz, A. et al. 2005, ApJ, 627, 29 
Ho, L. C. et al. 2011, ApJS, 197, 21

Keres, D. et al. 2005, MNRAS, 363, 2

Kennicutt, R. C. 1989, ApJ, 344, 685

Kormendy, J. \& Kennicutt, R. C. 2004, ARA\&A, 42, 603

Krumholz, M. \& Dekel, A. 2012, ApJ, 753, 16

Laine, J. et al. 2014, MNRAS, 441, 1992

Laurikainen, E. \& Salo, H. 2001, MNRAS, 324, 685

Le Borgne, J.-F. et al. 2004, A\&GA, 425, 881

Lilly, S. J. et al. 2013, ApJ, 882, 119

Lilly, S. J. et al. 1996, ApJL, 460, L1

Madau, P. et al. 1996, MNRAS, 283, 1388

Madau, P. \& Dickinson, M. 2014, ARA\&A, 52, 415

Malkan, M. et al. 1998, ApJS, 117, 25

Marino, R. A. et al. 2016, A\& A, 585, 47

Martini, P. \& Pogge, R. W. 1999, AJ, 118, 2646

Minchev, I. et al. 2012, A\& $A, 548,126$

Muñoz-Mateos, J. C. et al. 2015, ApJS, 219, 3

Noeske, K. G. et al. 2007, ApJL, 660, 43

Norman, C. et al. 1996, ApJ, 462, 114

Perez, I. 2004, A\& A, 427, 17

Pohlen, M. \& Trujillo, I. 2006, A\&A, 454, 759

Renzini, A. et al. 2015, ApJ, 801, 29

Roskar, R. et al. 2008a, ApJ, 675, 65

Roskar, R. et al. 2008b, ApJ, 684, 79

Roskar, R. et al. 2010, MNRAS, 408, 783

Sanchez, S. et al. 2012, A\&A A, 538, 8

Sanchez-Blazquez, P. et al. 2014, MNRAS, 437, 1534

Schaye, J. et al. 2010, MNRAS, 402, 1536

Seth, A. C. et al. 2008, ApJ, 678, 116

Sheth, K. et al. 2010, PASP, 122, 1397

Shlosman, I. \& Noguchi, M. 1993, ApJ, 414, 474

Strateva, I. et al. 2001, $A J, 122,1861$

Tacchella, S. et al. 2016, MNRAS, 452, 2790

van der Kruit, P. C. 1987, A\&A, 173, 59

Zhao-Yu, L. et al. 2011, ApJS, 197, 22 